RAE-IC, Revista de la Asociación Española de Investigación de la Comunicación

vol. 8, núm. 16 (2021), 29-50

ISSN 2341-2690

Recibido el 28 de junio de 2021

DOI: https://doi.org/10.24137/raeic.8.16.3

Aceptado el 1 de septiembre de 2021

\title{
Revisión de los estudios sobre el liderazgo y la autoría femenina en los medios: el caso de la prensa deportiva
}

Review of studies on female leadership and authorship in the media: the case of the sports press

Salido-Fernandez, Juana

Universidad de Granada (UGR)

juanasalido@correo.ugr.es

Forma de citar este artículo:

Salido-Fernandez, J. (2021). Revisión de los estudios sobre el liderazgo y la autoría femenina en los medios: el caso de la prensa deportiva. RAE-IC, Revista de la Asociación Española de Investigación de la Comunicación, 8(16), 29-50.

https://doi.org/10.24137/raeic.8.16.3

\section{Resumen:}

Las mujeres han aumentado en los últimos años su presencia en la profesión periodística, aunque las normas y estereotipos de género todavía mantienen muchos espacios de discriminación para estas, sobre todo en puestos de toma de decisiones, pero también en las propias redacciones, al limitarlas a unos ámbitos específicos acordes a su género. Este trabajo tiene como objetivo estudiar la situación de las mujeres en la producción informativa, centrándonos en un área tradicionalmente masculina como es la cobertura deportiva, para conocer si existen todavía hoy formas 
de discriminación hacia estas en el ejercicio de la profesión. Los resultados revelan que, la mayor cobertura del deporte femenino no se corresponde con un aumento de las firmas femeninas en tales medios. Las noticias deportivas continúan siendo cubiertas mayoritariamente por hombres, quienes además cuentan con mayor tiempo en antena, asumen funciones de mayor peso y copan los cargos de responsabilidad de los medios, mientras que las escasas periodistas presentes se ven restringidas a cubrir deportes tradicionalmente femeninos y perviven sesgos y estereotipos de género sobre ellas. Se discute sobre la exigua presencia de firmas femeninas en las redacciones deportivas y se proponen nuevos estudios que profundicen en las causas últimas de esta discriminación.

Palabras clave: comunicación, deporte, mujeres, estereotipos de género, periodismo deportivo, cobertura periodística, liderazgo femenino, autoría femenina, rutinas periodísticas.

\section{Abstract:}

Women have increased their presence in the journalistic profession in general in recent years, although gender norms and stereotypes still maintain many spaces of discrimination for women, especially in decision-making positions, but also in the newsrooms themselves, by limiting them to specific areas according to their gender. This work aims to study the presence of women in the journalistic profession, focusing on a traditionally male area such as sports coverage, to find out if there are forms of discrimination in the exercise of the profession. The results reveal that, despite the greater coverage of women's sports, this does not correspond to an increase in female signatures in these media. Sports news continue to be covered mostly by men, who also have more time on the air, assume more important functions and take over the positions of responsibility of these media, while the few existing female journalists are restricted to covering traditionally feminine sports and gender biases and roles persist about them. This meager presence of female firms in sports newsrooms is discussed and news studies are proposed that delve into the root causes of this discrimination. 
Keywords: communication, sport, women, gender stereotypes, sport press, media coverage, female leadership, female authorship, newsroom culture.

\section{INTRODUCCIÓN}

La práctica profesional en las redacciones de los medios está marcada por la cultura periodística, esto es, dinámicas laborales que establecen cómo se hacen las cosas siguiendo unas reglas, valores y prácticas que son aceptadas e interiorizadas hasta llegar a legitimar su ejercicio (North, 2016). Esta cultura periodística está, según Pedraza Bucio (2020), generizada, es decir, es una construcción marcada por el género, que se convierte en parte estructural de la construcción periodística. En este sentido, MelinHiggins, (2008, p. 46) encuentra que los hombres siempre han controlado las instituciones mediáticas, definiendo su organización, pero también cómo debe ser el periodismo. Esto supone no solo controlar los espacios periodísticos, sino también que lo masculino se convierta en el referente para el ejercicio de la profesión (Pedraza-Bucio, 2020). Pero a su vez, la cultura periodística niega esta influencia del género en la práctica profesional, mediante una apariencia de neutralidad que oculta jerarquías y posiciones que ocupan los sujetos. Lagarde (2005) aprecia en estos espacios elementos como la condición de género, referida a temas, formatos y fuentes entendidas como acordes a las mujeres, la posición de género, las situaciones que limitan la movilidad de estas en las estructuras de los medios, el puesto desempeñado o nivel de reconocimiento y la situación de género, referida a las posibilidades y restricciones que históricamente determinan la actividad periodística como las rutinas masculinas, remuneración desigual, acoso sexual...

Estas limitaciones que de forma general afectan a las mujeres, han ido superándose a lo largo de los años con el aumento de estas en la profesión, según muestran algunos informes como el Proyecto Global de Monitoreo de Medios (2020), que encuentra que el $40 \%$ de las personas que reportan y presentan las noticias tradicionales (prensa, radio y televisión) son mujeres y un $42 \%$ en las noticias en línea. Si comparamos con los datos del 2015, se aprecia un aumento del 3\% de mujeres reporteras en noticias impresas y 
televisivas, con cuatro de cada diez. Pero estos datos se deslucen si tenemos en cuenta criterios como la edad, pues a medida que esta aumenta, disminuye el número de mujeres presentadoras y reporteras, sobre todo estas últimas, no contabilizándose ninguna de más de 65 años y situándose entre los 19 y 34 años más del $60 \%$ de estas. También ocurre con los temas, ya que ellas se encargan, según este estudio, de cubrir en mayor medida temas sociales, de salud o género (45-55\%) mientras los hombres son más propensos a cubrir temas de política, economía o deporte (57-63\%). Ahondando en este último ámbito, la Encuesta Internacional de Prensa Deportiva, que estudió 80 periódicos en 22 países, encuentra que solo el $8 \%$ de más de 10.000 artículos fueron escritos por mujeres (Horky y Nieland, 2013). Tales datos provocan una falta de referentes femeninos a seguir en el ámbito de la comunicación deportiva, que hace que las jóvenes no la perciban como una carrera que puedan desarrollar o entiendan que resulta complicado para ellas alcanzar el éxito en este ámbito (Frank y O’Neill, 2016).

De este modo, este trabajo pretende realizar una revisión teórica sobre el estado de la cuestión de la autoría femenina en los medios deportivos. Para ello, se han extraído de las distintas bases de datos bibliográficas aquellos artículos que analizan la presencia femenina en los medios, con especial atención a los deportivos, tanto en el contexto internacional como en el caso español desde sus inicios hasta hoy. Este estudio proporcionará suficientes herramientas que permitirán examinar y desvelar la posición femenina actual en este espacio tan masculino, para problematizar sus consecuencias y plantear posibles propuestas de mejora.

\section{LAS MUJERES EN LA PRODUCCIÓN INFORMATIVA DE LOS MEDIOS}

Los primeros estudios sobre mujeres en los medios tenían como objetivo analizar cómo estas encajaban en las estructuras de producción de noticias, así como su infrarrepresentación y estereotipación en los contenidos y si este hecho afectaba a las audiencias (Lichter, Lichter y Rothman, 1986). A mediados de los noventa las periodistas alcanzaban el $27 \%$ de las redacciones de los medios, pero no superaban el $12 \%$ en los puestos ejecutivos, según un análisis llevado a cabo por Lünenborg (1996) en la prensa de referencia de nueve países europeos, afectando según este, a la imagen mostrada en 
los medios. La profesión periodística adquiere así formas masculinas, haciendo que la estructura de la redacción se organice en torno al hombre como modelo mientras las mujeres adquieren una posición subordinada (Byerly y Ross, 2003, p. 21).

La situación femenina en la producción de los medios no varía sustancialmente en los 2000, pues autores como Hannis y Strong (2007) encuentran en la prensa australiana un $29 \%$ de noticias escritas por las mujeres, con cierta mejora en la de Nueva Zelanda con el $37 \%$. Unos porcentajes que parecen retroceder cuando se supera la primera década del 2000, tal como muestra el estudio Women in Journalism (WIJ, 2011) sobre siete periódicos nacionales británicos durante un mes cualquiera, que encuentra un $78 \%$ de firmas masculinas, siendo el Daily Mail el diario con mayor paridad, con el 32\% de autoras femeninas, seguido de The Guardian con el $28 \%$ y, en último lugar, The Independent, con tan solo un 16\%. En los últimos años los investigadores siguen encontrando una escasa presencia femenina y una cultura de redacciones hegemónicamente masculina (Frank y O'Neill, 2016) que hace que los hombres tengas más probabilidades de ganar más dinero y alcanzar puestos de más responsabilidad (Barnes, 2017), un desequilibrio de género que se manifiesta a nivel mundial, tal como indica el informe preliminar del último Proyecto Global de Monitoreo (2020):

Las mujeres y las voces de las mujeres están sorprendentemente ausentes en la cobertura de noticias a nivel mundial. [...] La industria de las noticias debe adoptar códigos de conducta que definan y corrijan activamente la discriminación y los estereotipos de género, comenzando por un aumento en el liderazgo de las mujeres dentro de la industria de los medios (2020, p. 9).

En España autoras como Juana Gallego han estudiado a fondo los roles de género en la profesión periodística, encontrando que solo el $4,9 \%$ de la autoría periodística era femenina, mientras el $42,8 \%$ mostraba un punto de vista de ausencia total de género y un $47,6 \%$ era masculina. Este trabajo encontró que la pirámide jerárquica de los medios había aumentado la presencia de las periodistas al 22,7\%, aunque muy lejos todavía del 77,3\% masculino (2002, pp. 54-55), un incremento que no supuso para ellas ningún cambio cualitativo en el tratamiento de la información. Ana Jorge Alonso encuentra una situación similar en la cadena autonómica Canal Sur, y entiende que esto influye en la 
imagen mayoritariamente masculina que ofrece la cadena, independientemente del porcentaje de redactores y redactoras (2004). Según Gallego et al., (2002, p. 228) el género de quienes toman las decisiones sobre los contenidos es un factor más pero no el único ni el más importante, puesto que influyen otros aspectos como las propias estructuras de las empresas informativas, la cultura periodística y rutinas informativas, formada por las creencias y valores compartidos por la profesión y que sustentan su credibilidad, el contexto social y cultural en el que se desarrollan las noticias o la idiosincrasia y esquemas ideológicos del propio periodista. Además, los medios se preocupan por adaptar sus contenidos al espacio del que disponen, a sus audiencias y a la competencia, sin contar con que las agencias de noticias, gabinetes y empresas nutren a estos de informaciones que son empaquetadas y difundidas de forma genérica (Gallego et al., 2002). Por todo esto, Plaza y Delgado (2007, p. 185) perciben a los periodistas como parte del engranaje del contexto mediático imperante, donde se incluyen conglomerados económicos y políticos, que, como toda gran estructura, mantienen el orden establecido por las élites dominantes, que controlan lo que se dice, lo que se neutraliza o que se prejuzguen temas considerados diferentes, como son las cuestiones de género. En este sentido Diezhandino destaca que:

Los medios feminizaron sus redacciones, pero el acento de sus contenidos sigue siendo masculino. Lo cual es sintomático de que la mujer periodista ha asumido los valores y criterios que marca toda una tradición de imperativo masculino. Una voz parece hacerse más creíble si procede de un varón. Sólo el cargo permitirá que hablen las mujeres (2008, p. 23).

En esta línea Tamarit, Quintana y Plaza (2011, p. 259) entienden que "las rutinas profesionales de los periodistas son un freno para facilitar la visibilidad de las mujeres en el ámbito público y no se perciben diferencias cuando la información es escrita por un hombre o una mujer". Pero, además, cuando la protagonista de la información es una mujer, se aprecia un exceso de confianza al citarla, mediante nombres de pila o identificándola por el apellido del esposo o por su parentesco con un hombre (MuñozMuñoz y Salido-Fernandez, 2018) lo que supone un freno para que puedan ocupar la presencia y respeto que les corresponde (Tamarit, Quintana y Plaza, 2011). Por su parte, 
Mateos de Cabo (2007), también aprecia que los hombres disfrutan de prioridad informativa mientras las mujeres deben asumir roles secundarios, y defiende que un mayor número de mujeres periodistas pueden traer consigo más presencia femenina en las noticias puesto que "las mujeres mencionan a más mujeres que los hombres. [...]. La presencia de mujeres en los cargos de responsabilidad de las redacciones influye positivamente en el porcentaje de mujeres que aparecen en las noticias" (2007, p. 14). En este sentido el Proyecto Global de Monitoreo (2020) confirma que las mujeres reporteras en mayor probabilidad que los hombres recurren a sujetos y fuentes femeninas (31\% frente al 24\%). Por otro lado, junto a la escasa presencia, según Menéndez (2007) existe una especialización de las mujeres en áreas "femeninas", que se corresponden con las secciones "blandas", como son sociedad o cultura, que carecen del prestigio profesional de las "duras", y además son discriminadas por razón de sexo en la promoción profesional. Unos problemas que se amplían debido a la desigualdad en los cargos de responsabilidad y de toma de decisiones, al contar con menor confianza por parte de los jefes, lo que se traduce en peor remuneración, menores expectativas y opciones de ascender en el organigrama, así como desigual uso de espacios, rutinas masculinas en los horarios, e incluso, acoso y abuso sexual (Vega Montiel, 2010).

Bien es cierto que en los últimos años las periodistas han aumentado en número y posición en los medios y han ido mostrándose contenidos sobre mujeres cada vez más variados, pero esto no ha impedido que hayan tenido que adaptarse al orden de género dominante, tanto en los contenidos como en las estructuras de las empresas mediáticas, puesto que "las mujeres en los medios han padecido de la misma inequidad que las mujeres de los medios" (Pedraza Bucio, 2012, p. 55).

Si examinamos la situación de los últimos años, el predominio de ideologías patriarcales, relativas al conjunto de creencias que constituyen la base de las diferencias entre hombres y mujeres (Facio y Fríes, 2005), no ha disminuido en los medios, pues Navarro y Mac Donald (2016) en su estudio de los informativos del Canal 9 valenciano, encuentran un preocupante discurso sexista en la prensa, como no nombrar a las mujeres por sus nombres y apellidos, disonancias gramaticales, perfiles de poder (expertos, políticos, científicos...) solo masculinos frente a una representación femenina 
de inexpertas o víctimas, asociadas a lo doméstico y emocional. Una situación que también se expande a los medios digitales, donde no se aprecian cambios con respecto a los tradicionales, pues las mujeres representan el $41 \%$ de autoras en estos (GMMP, 2020). También Soria Ibáñez (2016) aprecia que la firma masculina prevalece sobre la femenina, algo que se prolonga a los puestos de dirección, lo que puede afectar para seguir un modelo de periodismo no sexista, ya que, junto al androcentrismo de las redacciones, también existe una carencia de formación en igualdad de las empresas periodísticas en España.

\section{LAS MUJERES EN EL ESPACIO MEDIÁTICO DEPORTIVO}

La presencia de las mujeres en el espacio público de construcción cultural está todavía marcada por los roles y estereotipos que las asocian a lo doméstico y privado, más si cabe cuando este espacio es un ámbito simbólico masculino como ocurre con el deporte y la prensa deportiva, lo que se traduce en una doble exclusión para ellas (PedrazaBucio, 2012), pues sus voces están marginadas en el periodismo en general, tal como hemos visto, pero todavía más en secciones prestigiosas como son la política, economía o los deportes (Gallego, 2002). En este último ámbito Capranica et al., (2005, p. 213) encuentran que el dominio y hegemonía masculinos contribuyen a mantener la idea de que el deporte es un espacio de hombres, pues las normas de género tradicionales impactan en las percepciones de la audiencia sobre el papel femenino en la sociedad, algo que en el deporte es muy notorio debido al sexismo imperante en este (Fink, 2016). Así, los periodistas transmiten unos marcos conceptuales que actúan como esquema de interpretación para las audiencias (Goffman, 1974, p. 21), y estos marcos mantienen sesgos de género, algo que para Sartore y Cunningham (2007) incita a las mujeres a renunciar a sus metas y autolimitarse en este espacio eminentemente masculino, haciendo a su vez que los contenidos se adecuen al sexo masculino. Los periodistas deportivos construyen un sentido de la realidad que es culturalmente codificado y se basa por lo general en ideologías patriarcales, de modo que sus prejuicios sobre el deporte pueden ser reproducidos consciente e inconscientemente, lo que supone que el discurso sexista que transmiten sea difundido a millones de espectadores, consiguiendo un gran alcance social (Rojas Torrijos, 2010). 


\subsection{EL LIMITADO ACCESO DE LAS PERIODISTAS A LA PRENSA DEPORTIVA}

Las mujeres comienzan a incorporarse a la sección de deportes de los medios en la década de los 20 del siglo pasado, aunque no sería hasta los 60 cuando empiezan a denunciar el desequilibrio existente, y se crean organismos como la Association for Women in Sports Media (1987), destinada a apoyar la presencia femenina en los medios deportivos y favorecer una representación justa, diversa y positiva, así como un acceso más equitativo. A pesar de estas reivindicaciones iniciales, años después encontramos que las redacciones deportivas siguen siendo mayoritariamente masculinas, pensadas por hombres y para los hombres, tal como revelan Hardin y Shain (2007) en un estudio que analiza los 200 periódicos de mayor difusión, donde las mujeres alcanzan un 11\% como periodistas deportivas, aunque en secciones generales esta cifra asciende al 39\%.

En esta línea, la International Sport Press Survey 2011 (Horky y Nieland, 2013) reportó que el $92 \%$ de las informaciones firmadas en la prensa deportiva estaban escritas por hombres, y Franks y O'Neill (2014) encuentran un desequilibrio todavía mayor hacia las periodistas deportivas en la prensa británica, pues solo el $2 \%$ de los artículos estaban firmados por mujeres. Similares fueron los datos aportados en un análisis de los mayores periódicos de EEUU realizado por Harp, Bachmann y Loke (2014), que revela que solo el $4,5 \%$ de las noticias sobre deportes estaban escritas por mujeres, algo que Cooky, Messner y Musto (2015) aplican en la televisión, encontrando en la cadena deportiva ESPN que solo el 4,4\% de presentadores y copresentadores eran mujeres.

En España encontramos varios estudios en esta línea, como el realizado por Martín y Amurrio (2007) sobre los perfiles de los periodistas deportivos en los medios informativos audiovisuales del País Vasco, que reportaron grandes diferencias entre sexo y edad, pues las mujeres en un $65 \%$ se situaban entre los 20 y 25 años y a medida que avanzaban de edad su presencia iba disminuyendo de forma drástica, lo que indica que en la prensa deportiva se agravan las limitaciones de edad ya percibidas en la prensa general. También el Consejo Audiovisual de Andalucía (2008), en un estudio sobre género y televisión en las redacciones, aprecia un $20 \%$ de mujeres entre los reporteros, enviados especiales y locutores de eventos deportivos, mientras que los hombres 
alcanzan el $80 \%$ y en su análisis longitudinal de la prensa deportiva, Sainz de Baranda (2013) revela que las mujeres representan solo el 9,1\% del total de las firmas de las noticias femeninas.

Los estudios se van ampliando a nuevos formatos y medios para medir el nivel de desigualdad, y encontramos que según el informe sobre género y raza llevado a cabo por la Associated Press Sports Editors (APSE) en 2014, las mujeres representaban apenas el 13,3\% del personal de periódicos y webs deportivas (Lapchick y Baker, 2016), con un $12,7 \%$ de reporteras, otro $12,4 \%$ de columnistas y el $9,9 \%$ de editoras, evidenciando que cuanto más alto fuera el puesto desempeñado, menos probabilidades había de que fuese ocupado por una mujer. Muy en línea con estos datos, la Women's Media Center (2017) encuentra que las mujeres continúan muy por detrás de los hombres en las redacciones deportivas en países como EEUU, con un porcentaje de apenas el $11 \%$.

Esta desigualdad alcanza también grandes eventos como los Juegos Olímpicos, donde aunque existe un notable avance en la cobertura deportiva femenina a nivel cuantitativo (Salido-Fernandez y Muñoz-Muñoz, 2021a), debido a un esfuerzo de las instituciones por lograr una participación más equilibrada y por el carácter nacionalista del evento, que otorga mayor igualdad en la cobertura, ya que lo importante es conseguir medallas y no tanto el sexo de quien la consigue (Capranica et al., 2005, Salido-Fernandez, 2020), esto no se hace extensivo a la producción de noticias, con mayores desequilibrios. En este sentido, Coche y Tuggle (2018), encontraron que la NBC ofreció para los Juegos de Londres a comentaristas, periodistas y reporteros masculinos una presencia del $84 \%$ frente a solo el $16 \%$ femenino, siendo estas además ex deportistas contratadas por el medio y no periodistas, y con mucha menos diversidad en la cobertura que los hombres, pues se encargaban de cubrir principalmente los eventos femeninos, a diferencia de los hombres que cubrían ambos (Salido-Fernandez y Muñoz-Muñoz, 2021b). Algunos autores como North (2016) entienden que la marginación de las periodistas deportivas responde a la misma lógica que de las atletas, pues opera la misma cultura de redacción que ignora a las mujeres frente a los varones.

\subsection{PRESENCIA DE SESGOS Y ROLES DE GÉNERO EN LOS MEDIOS DEPORTIVOS}


Muchos autores han profundizado en la forma en que se presenta el desequilibrio en su vertiente cualitativa, así Melin-Higgins (2008) estudia a través de entrevistas el comportamiento de las periodistas deportivas, y encontró que estas salvaban la contradicción que representaban, es decir, ser reconocidas profesionalmente "a pesar de ser mujeres", anulando en cierta forma su condición de mujeres, mimetizándose con las formas y reglas propias masculina, esto es, siguiendo la estrategia de ser "una de ellos", lo que para Schmidt (2015) es entrar en el "Boys Club" del periodismo. Esto supone interiorizar presupuestos tales como narrar los acontecimientos a través de la dicotomía público/masculino-privado/femenino, mantener las distancias para ser profesional, creer que lo que se emite es siempre la información más relevante y no una perspectiva más del acontecimiento o que informar en masculino genérico es lo objetivo y lo subjetivo es hablar de la realidad de las mujeres (Melin-Higgnins, 2008). Por todo esto, muchas periodistas temen ser acusadas de sesgo en favor de las atletas femeninas, y de ser percibidas como menos profesionales o egoístas (Kian, 2007), lo que hace que las voces femeninas sean relegadas, a no ser que adopten en su práctica diaria una lógica dominante. Así, como mujeres, se les presupone que deben ser femeninas, empáticas, comprensivas y educadas, pero a la vez, han de negar estas cualidades y combatir su feminidad y enfoque culturalmente enraizado si quieren ser consideradas buenas profesionales (Hardin y Shain, 2007).

Por otro lado, si atendemos a las disciplinas, los roles tradicionales de género son de nuevo protagonistas, así deportes como el boxeo, fútbol o motor están sumamente masculinizados y son más prestigiosos, mientras que a las periodistas se les asignan historias más "suaves" y deportes considerados apropiados para ellas como gimnasia, patinaje o tenis (Pedraza Bucio, 2012). Así, Kian y Hardin (2009) encontraron que las periodistas eran más propensas a cubrir el baloncesto femenino mientras que los varones cubrían ambos, y en cuanto al tratamiento, ellos tenían más probabilidades de reforzar estereotipos de género al elogiar más el carácter atlético masculino mientras ellas destacaban más la destreza atlética femenina. Esto supuso para estos autores entender que la presencia de periodistas mujeres podría marcar las diferencias en función del encuadre mediático, al estar dispuestas a desafiar la hegemonía masculina, 
aunque de forma sutil pero significativa, puesto que las estructuras institucionales pueden minimizar este impacto.

Es interesante profundizar en este aspecto, pues aunque es cierto que algunos estudios vinculan la mayor presencia de periodistas deportivas con una mayor cantidad de informaciones sobre deporte femenino, otros entienden que la complejidad del proceso de producción de noticias, las demandas de la organización y la toma de decisiones de los editores, las rutinas de trabajo, las percepciones del interés de la audiencia, e incluso, las propias actitudes, son factores que pueden influir mucho más en que se reduzca o trivialice la cobertura femenina en el deporte (Kian, 2007). En este sentido Boyle, Millington y Vertinsky (2007) señalan directamente a la cultura machista propia de la sección de deportes como una de las culpables de la escasa presencia de periodistas para cubrir disciplinas como el fútbol, pues esta cultura entiende que las mujeres no tienen un conocimiento apropiado para comentar y analizar este.

A este respecto, Eagly y Karau (2002) quisieron conocer la credibilidad de las periodistas deportivas y encontraron que todavía hoy los roles que ellas desempeñan en la industria de los medios son escasos y asociados a su género, lo que mantiene sesgos y sexismo que afecta a su credibilidad ante la audiencia. Estos hallazgos responden a la teoría del rol social, según la cual, si un individuo lleva a cabo una actividad distinta a lo asociado tradicionalmente a su género, es percibida como una incongruencia a sus roles de género (Eagly y Karau, 2002). Mudrick et al., (2016) relacionan esta teoría con que los hombres sigan dominando los roles de mayor peso en los medios deportivos, al reforzar estereotipos grupales de que son más apropiados para esta labor, ya que tienen mayor conocimiento sobre el deporte, excluyendo a las mujeres de estas secciones (Hardin y Shain, 2007) porque son percibidas con menor conocimiento, y por tanto, con menor credibilidad que sus colegas masculinos (Etling y Young, 2007), así como menor autoridad, entendida siguiendo el concepto de dominación tradicional de Weber, propia aunque no exclusiva de la era patriarcal (Cataño, 2000).

Schmidt (2018) encuentra que los periodistas masculinos son significativamente menos propensos que las periodistas a mostrar su apoyo a un mayor papel de las mujeres, 
además estos cuentan con más tiempo en los medios (Gallego et al., 2002) y generan más comentarios sobre los deportistas masculinos. De hecho, aunque las mujeres sean comentaristas deportivas encuentran resistencias significativas en la industria y reciben un escrutinio negativo del público (Grubb y Billiot, 2010). Así, Davis y Krawczyk (2010) encontraron que las mujeres periodistas eran percibidas de forma positiva por las audiencias en cuanto a experiencia y profesionalidad, pero no generaban autenticidad o credibilidad en las transmisiones en televisión.

Además, las periodistas son comparadas constantemente con sus colegas varones y se les exige mucho más que a ellos, según explica Lagarde (2005), pues cualquier error en una periodista supone un desprestigio para todo el colectivo femenino, asociado en gran medida a la falta de conocimiento o eficacia.

\subsection{LA PRENSA DEPORTIVA COMO ESPACIO DE SEXUALIZACIÓN Y ACOSO FEMENINO}

La apariencia física también ofrece percepciones diferenciadas de cada sexo para la audiencia, según han estudiado autores como Etling y Young (2007), que midieron el sesgo sexual en la autoridad de los reporteros, y los varones mostraron un nivel más alto de autoridad. Davis y Krawczyk (2010) realizaron un estudio similar y encontraron que el atractivo se correlaciona positivamente con la competencia, pericia, dinamismo y confiabilidad, excepto si las periodistas eran muy atractivas, en cuyo caso recibieron reacciones violentas por su apariencia. Ambos estudios revelaron que los hombres eran vistos como más competentes, con más experiencia y más persuasivos para las audiencias que las mujeres.

Además, en una profesión abrumadoramente masculina, especialmente en la cobertura de deportes como el fútbol, las periodistas no suelen conducir ningún programa, sino que solo consiguen ser colaboradoras, muchas veces a pie de campo, para lo que deben cumplir con unos requisitos más físicos que profesionales:

La estructura del periodismo deportivo ha condicionado la incorporación de las mujeres a la labor de comentaristas en la medida en que cumplan con un ideal estético, útil para embellecer cualquier producción. [...] condicionar a las comentaristas y reporteras de deportes a la reproducción de un ideal estético reduce su ejercicio 
profesional del periodismo, porque no se prioriza su capacidad sino su belleza (Pedraza Bucio, 2012, p. 58).

De hecho, estas reporteras llegan a encabezar rankings de mujeres más atractivas (Cooky, Messner y Musto, 2015), lo que hace que sean objetualizadas para atraer más audiencia y que sus cuerpos reciban mucha más atención que los de sus colegas (Cummins et al., 2019). Además, el hecho de que las reporteras y presentadoras deportivas sean muy jóvenes y atractivas, supone mantener una severa subrepresentación de las de mayor edad (GMMP, 2020).

Un posible efecto de esta sexualización de las periodistas es que sean acosadas y desafiadas por aficionados deportivos (Grubb y Billiot, 2010), puesto que un mayor acceso a las noticias digitales, también supone un mayor contacto con los profesionales de los medios, que resultan más accesibles a través de sus perfiles en redes sociales, correo electrónico... de modo que los posibles castigos de la audiencia y los sesgos en la contratación, afectan a las mujeres en la industria deportiva pero además, afectan a la percepción social sobre cuáles son las ocupaciones que debe tener una mujer en sociedad. Everbach (2018) encuentra que las mujeres periodistas reconocen que las redes han mejorado su trabajo, pero también han dado pie al fomento de acosadores, al convertirse en un foro donde llevar a cabo juicios públicos y privados que pueden causar daños duraderos en la salud y bienestar de las periodistas. En este sentido, una encuesta realizada en 2016 a las periodistas de radio, revela que estas pensaban que las redes sociales habían aumentado las críticas de los espectadores sobre su apariencia (Fieneman y Jenkins, 2018).

\section{CONCLUSIONES}

Las mujeres en el periodismo deportivo continúan en una situación de escasez y desequilibrio con respecto a sus colegas masculinos. Algo que ocurre por la limitada presencia que estas tienen en las redacciones y puestos de responsabilidad de las empresas informativa (Gallego, 2002; Hardin y Shain, 2007; Frank y O'Neil, 2014), incluyendo durante grandes eventos como los Juegos Olímpicos (Capranica et al., 2005; Coche y Tuggle, 2018), y sin importar el país estudiado, EEUU (Hardin y Shain, 2007), 
Reino Unido (Franks y O’Neill, 2014) o España (Gallego, 2003; Jorge Alonso, 2004, Sainz de Baranda, 2013). Pero junto a esta escasa presencia se suman otras diferencias cualitativas tales como la falta de credibilidad y autoridad que se les atribuye por parte de las audiencias (Eagly y Karau, 2002; Etling y Young, 2007, Mudrick et al, 2016), pero también el relegarlas a áreas menos prestigiosas y deportes considerados apropiados femeninos (Kian y Kardin, 2009). Además, se fomenta una sexualización de las periodistas, que han de cumplir con unos requisitos estéticos de juventud y belleza para poder aparecer en los medios (Cooky, Messner y Musto, 2015; Cummins et al, 2019), lo que hace que reciban más atención por su físico que por su labor profesional y sean objetualizada y, en muchos casos, también acosadas por el público (Grubb y Billiot, 2010, Fink, 2016; Thomas y Jenking, 2019).

En definitiva, las pocas mujeres que consiguen acceder a una profesión tan masculina como es el periodismo deportivo, deben hacerlo con unas limitaciones y requisitos muy estrictos, que impiden que estas puedan llegar a convertirse en referentes habituales para las jóvenes y niñas, que encontrarán aquí un espacio de hegemonía masculina que perpetúa los desequilibrios existentes. Para ello, es fundamental que las industrias mediáticas del deporte pongan en marcha acciones encaminadas a erradicar esta desigualdad, pues solo favoreciendo una mayor participación de mujeres en todos los escalones de la cadena de producción de noticias y un reparto equilibrado de las secciones y disciplinas, especialmente en áreas de mayor dominio masculino y sin restricciones de edad o requisitos físicos, se podrá normalizar la presencia y labor de las mujeres en este ámbito. Además, esta mayor presencia de firmas femeninas ampliará, tanto en cantidad como en enfoque, el equilibrio de género en los contenidos de estos medios. Todo ello, debe combinarse con la elaboración desde las instituciones y empresas mediáticas de guías y libros de estilo que promuevan una información no sexista, y sean útiles, accesibles y de obligado cumplimiento por los medios, junto con una formación con perspectiva de género para los futuros periodistas. Por último, se propone profundizar en nuevos estudios, especialmente en el caso español, que analicen la estructura de los medios para descubrir si guarda relación con el tipo de contenidos que estos publican, con especial atención a los nuevos formatos digitales, a 
través de entrevistas en profundidad y encuestas a los profesionales. Pero, además, la investigación debe ampliarse a deportes menos conocidos o de ámbito local, así como a otros espacios de comunicación tradicionalmente masculinos como las secciones de política, economía o ciencia, para desvelar si existen desequilibrios tanto en sus redacciones como en sus contenidos y analizar si un incremento de mujeres en la estructura informativa de estas, se traduciría en una mayor igualdad en tales contenidos, tal como revelan los estudios analizados.

\section{REFERENCIAS BIBLIOGRÁFICAS}

Asociación Mundial para la Comunicación Cristiana (2020). Informe preliminar GMMP: ¿Qué avances hay para la igualdad de género en las noticias 25 años después de Beijing? Recuperado de https://www.uv.mx/blogs/obvio/gmmp/informe-preliminargmmp2020/

Barnes, L. (2017). An inexplicable gap: Journalism and gender in New Zealand. Journalism, 18(6), 736-753. https://doi.org/10.1177/1464884915620231

Boyle, E., Millington, B. y Vertinsky, P. (2006). Representing the Female Pugilist: Narratives of Race, Gender and Disability in Million Dollar Baby. Sociology of Sport Journal, 23(29), 99-116. https://doi.org/10.1123/ssj.23.2.99

Byerly, M. y Ross, K. (2003). Women \& Media. Reino Unido: Blackwell Publishing. Capranica, L., Mingati, C., Billat, V., Hanghoj, S., Piacentini, M. F., Cumps, E. y Meeusen, R. (2005). Newspaper coverage of women's sports during the 2000 Sydney Olympic Games: Belgium, Denmark, France, and Italy. Research Quarterly for Exercise and Sport, 76(2), 212-223. https://doi.org/10.1080/02701367.2005.10599282

Cataño, G. (2000). Educación y formas de dominación en Max Weber. Revista Colombiana de Educación, 40-41. https://doi.org/10.17227/01203916.7773

Coche, R. y Tuggle, C. A. (2018). Men or women, only five Olympic sports matter: A quantitative analysis of NBC's prime-time coverage of the Rio Olympics. Electronic News, 12(4), 199-217. https://doi.org/10.1177/1931243117739061 
Consejo Audiovisual de Andalucía (2008). Estudio sobre género y deporte en televisión. Recuperado de https://cutt.ly/aEzR7kF

Cooky, C., Messner, M. y Musto, M. (2015). It's Dude Time! A Quarter Century of Excluding Women's Sports in Televised News and Highlight Shows. Communication \& Sport, 3(3), 261-287. https://doi.org/10.1177/2167479515588761

Cummins, R. G., Ortiz, M. y Rankine, A. (2019). "Elevator Eyes" in Sport Broadcasting: Visual objectification of Male and Female Sports Reporters. Communication \& Sport, 7(6), 1-22. https://doi.org/10.1177/2167479518806168

Davis, D. C. y Krawczyk, J. (2010). Female sportscaster credibility: Has appearance taken precedence? Journal of Sports Media, 5(2), 1-34. 10.1353/jsm.2010.0004

Diezhandino, M. P. (Dir.). (2008). El Periodismo en la era de Internet. Claves para entender la situación de la información periodística en España. Colección Fundación Telefónica. Madrid: Ariel.

Eagly, A. y Karau, S. J. (2002). Role congruity theory of prejudice toward female leaders. Psychological Review, 109(3), 573-598. https://doi.org/10.1037/0033$295 x .109 .3 .573$

Etling, L. y Young, R. (2007). Sexism and the authoritativeness of female sportscasters. Communication Research Report, 24(2), 121-130

https://doi.org/10.1080/08824090701304816

Everbach, T. (2018). "I realized it was about them...not me". Women sports journalists and harassment. En J. Vickery y T. Everbach (Eds.) Mediating misogyny, (pp. 131-149). https://doi.org/10.1007/978-3-319-72917-6_7

Facio, A. y Fríes, L. (2005). Feminismo, Género y Patriarcado. Academia. Revista sobre Enseñanza del Derecho de Buenos Aires, 6, 259-294. 
Fienneman, T. y Jenkins, J. (2018). Sexism on the Set: Gendered Expectations of TV Broadcasters in a Social Media World. Journal of Broadcasting \& Electronic Media, 62(3), 479-494. https://doi.org/10.1080/08838151.2018.1484292

Fink, J. (2016). Hiding in Plain Sight: The embedded Nature of Sexism in Sport. Journal of Sport Management, 30(1), 1-7. https://doi.org/10.1123/jsm.2015-0278

Frank, S. y O’Neill, D. (2016). Women reporting sport: Still a man's game? Journalism, 17(4), 474-492. https://doi.org/10.1177/1464884914561573

Gallego, J. (2002). La prensa por dentro. Producción informativa y transmisión de estereotipos de género. Comunicación y Sociedad, 16(2), 49-66. Recuperado de https://dadun.unav.edu/bitstream/10171/8055/1/20091008140241.pdf

Gallego, J., Altés, E., Melús, M. E., Soriano, J. y Cantón, M. J. (2002). La prensa diaria por dentro: mecanismos de transmisión de estereotipos de género en la prensa de información general. Anàlisi: quaderns de comunicació i cultura, 28, 225-242. Recuperado de https://www.raco.cat/index.php/Analisi/article/view/15110/14951

Goffman, E. (1974). Frame analysis: An essay on the organization of experience. Cambridge: Harvard University Press.

Grubb, M. V. y Billiot, T. (2010). Women sportscasters: Navigating a masculine domain. Journal of Gender Studies, 19(1), 87-93. https://doi.org/10.1080/09589230903525460

Hardin, M. y Shain, S. (2007). Feeling much smaller than you know you are. The fragmented professional identity of female sports journalists. Critical Studies in Media Communication, 23(4), 322-338. https://doi.org/10.1080/07393180600933147

Harp, D., Bachmann, I. y Loke, J. (2014). Where are the Women? The presence of Female Columnists in U.S Opinion Pages. Journalism \& Mass Communication Quarterly, 91(2), 289-307. https://doi.org/10.1177/1077699014527457

Horky, T. y Nieland, J.U. (2013). International sports Press Survey 2011- Quantity and Quality of Sports Reporting. Nordest: Book son Demand GmbH. 
Jorge-Alonso, A. (2004). Mujeres en los medios, mujeres de los medios: imagen y presencia femenina en las televisiones públicas: Canal Sur TV. Málaga: Icaria Editorial.

Kian, E. M. (2007). Gender in sport writing by the print media: An exploratory examination of writers experiences and attitudes. The SMART Journal 4(1), 5-26 Recuperado de http://thesmartjournal.com/genderinsw.pdf

Kian, E. M. y Hardin, M. (2009). Framing of sport coverage based on the sex of sports writers: Female journalists counter the traditional gendering of media coverage. International Journal of Sport Communication, 2(2), 185-204. https://doi.org/10.1123/ijsc.2.2.185

Lagarde, M. (2005). Género y feminismo. Desarrollo Humano y Democrático. Madrid: horas y HORAS.

Lapckick, R. y Baker, D. (2016). The 2015 racial and gender report card: National Basketball Association. Orlando: The institute for Diversity and Ethics in Sport.

Lichter, S. R., Lichter, L. S. y Rothman, S. (1986). The politics of the American dream From Lucy to Lacey: TV's Dream Girls. Public Opinion, 9(3), 16-19.

Lünenborg, M. (1996). Journalists in Europe: an international comparative study. Wiesbaden: Westdeutscher Verlag.

Martín, R. M. y Amurrio, M. (2007). Periodistas del medio audiovisual: formación y situación laboral. En C. Peñafiel (Ed.), Transformaciones de la radio y la televisión en Europa (pp. 119-213). Zarautz: Servicio editorial de la Universidad del País Vasco.

Mateos-de-Cabo, R. (Coord.). (2007). La presencia de estereotipos en los medios de comunicación: análisis de la prensa digital española. Madrid: Dirección General de la Mujer, Comunidad de Madrid. Recuperado de http://goo.gl/rxuiQW

Melin-Higgins, M. (2008). Gendered Journalism Cultures. Malmo: University of Göteborg. 
Menéndez, M. I. (2007). Una representación invisible: imagen actual de las mujeres en la información. En Mujeres en medio: repaso crítico a los medios de comunicación y su lenguaje (pp. 77-96). Asociación de Mujeres Profesionales de la Comunicación (AMECO).

Mudrick, M., Miller, M. y Atkin, D. (2016). The influence of social media on fan reactionary behaviors. Telematics and Informatics, 33(4), 896-903.

https://doi.org/10.1016/j.tele.2016.01.005

Muñoz-Muñoz, A. M. y Salido-Fernandez, J. (2018). Tratamiento informativo de las esposas y novias (WAGs) de los deportistas en la prensa deportiva digital española. EI profesional de la información, 27(2), 331-340.

https://doi.org/10.3145/epi.2018.mar.11

Navarro, I. y Mac-Donald, P. (2016). La infrarrepresentación de la mujer en los informativos de Canal 9-RTVV. E-Aesla, 2, 224-235. Recuperado de http://hdl.handle.net/10251/99605

North, L. (2016). The Gender of "soft" and "hard" news: Female journalists' views on gendered story allocations. Journalism Studies, 17(3), 356-373.

https://doi.org/10.1080/1461670X.2014.987551

Pedraza-Bucio, C. (2012). Mujeres en el periodismo deportivo: reflexiones para comprender la transgresión desde la práctica discursiva de las reporteras de deportes. Derecho a comunicar, 4, 47-69. Recuperado de https://biblat.unam.mx/hevila/Derechoacomunicar/2012/no4/4.pdf

Pedraza-Bucio, C. (2020). El mito de la cancha neutral: la asignación generizada en las redacciones de la prensa deportiva. Revista interdisciplinaria de estudios de género de El Colegio De México, 6(1), 1-31. https://doi.org/10.24201/reg.v6i0.510

Plaza, J. F. y Delgado, C. (2007). Género y comunicación. Madrid: Editorial Fundamentos. 
Rojas-Torrijos, J. L. (2010). La construcción de las noticias deportivas desde una mirada androcéntrica. De la invisibilidad a los estereotipos de la mujer deportista. Revista de Comunicación Vivat Academia, 113, 122-136.

https://doi.org/10.15178/va.2010.113.122-136

Sainz de Baranda, C. (2013). Mujeres y deporte en los medios de comunicación. Estudio de la prensa deportiva española (1979-2010). Universidad Carlos III de Madrid, Madrid. Recuperado de https://e-archivo.uc3m.es/handle/10016/16505\#preview

Salido-Fernandez, J. (2020). Olimpismo, género y comunicación. Una aproximación al deporte femenino y a su representación en los medios deportivos. Comunicación y género 3(2), 173-182. https://doi.org/10.5209/cgen.68559

Salido-Fernandez, J. y Muñoz-Muñoz, A. M. (2021a). Representación mediática de las deportistas en los Juegos Olímpicos: una revisión sistemática. Apunts. Educación Física y Deportes, 146, 32-41. https://doi.org/10.5672/apunts.2014-0983.es.(2021/4).146.04

Salido-Fernandez, J. y Muñoz-Muñoz, A. M. (2021b). Men's and women's basketball coverage in the Spanish digital press during the 2016 Rio Olympics. Journalism Practice (en prensa).

Sartore, M. L. y Cunningham, G. B. (2007). Explaining the under-representation of women in leadership positions of sport organizations: A symbolic interactionist perspective. Quest, 59(2), 244-265. https://doi.org/10.1080/00336297.2007.10483551 Schmidt, H. C. (2015). "Still a boy club". Perspectives on female sports and sports reporters in university student newspapers. Qualitative Research Reports in Communication, 16(1), 65-74. https://doi.org/10.1080/17459435.2015.1086422 Schmidt, H. C. (2018). Forgotten Athletes and Token Reporters: Analyzing the Gender Bias in Sports Journalism. Atlantic Journal of Communication, 26(1), 59-74. https://doi.org/10.1080/15456870.2018.1398014 
Soria Ibáñez, M. M. (2016). Noticias con perspectiva de género: la visión de os periódicos de referencia nacional. Revista De La Asociación Española De Investigación De La Comunicación, 3(5), 75-81. https://doi.org/10.24137/raeic.3.5.10

Tamarit, A., Quintana, N. y Plaza, J. F. (2011). ¿Quién habla de las mujeres en las noticias donde ellas son las protagonistas? Palabra clave, 14(2), 247-260. Recuperado de http://www.scielo.org.co/scielo.php?script=sci_abstract\&pid=S0122$82852011000200005 \& \operatorname{lng}=e n \& n r m=i s o \& \operatorname{lng}=e n$

Vega-Montiel, A. (2010). Las mujeres y el derecho humano a la comunicación: su acceso y participación en la industria mediática. Revista mexicana de ciencias políticas y sociales, 52 (208), 81-95. Recuperado de http://www.scielo.org.mx/scielo.php?script=sci_arttext\&pid=S0185$19182010000100005 \& \operatorname{lng}=e s \&$ tlng=es.

Women in Journalism (2011). Recuperado de https://womeninjournalism.co.uk/research/ Women's Media Center (2017). Divided 2017: The Media Gender Gap. Recuperado de https://www.womensmediacenter.com/reports/divided-2017 